\title{
Image Analysis: Is the Fourier Transform Becoming Obsolete?
}

\author{
Noël Bonnet $\left({ }^{1,2}\right)$ and Philippe Vautrot $\left({ }^{2}\right)$ \\ $\left.{ }^{1}\right)$ Unité INSERM 314 (IFR 53 Biomolécules), 21 rue Clément Ader, 51685 Reims Cedex 2, \\ France \\ $\left({ }^{2}\right)$ Université de Reims, 21 rue Clément Ader, 51685 Reims Cedex 2, France
}

(Received October 15; accepted November 15, 1996)

\author{
PACS.02.30.-f - Function theory, analysis \\ PACS.02.70.-c - Computational techniques \\ PACS.02.30.Px - Abstract harmonic analysis \\ PACS.42.30.Kq - Fourier optics \\ PACS.42.30.Yc - Other topics in imaging and optical processing
}

\begin{abstract}
We show that, despite its great usefulness in image processing and analysis, the Fourier transform may also suffer from certain drawbacks and limitations. We show how several limitations of a technical nature can be overcome. More importantly, we show that the fundamental limitation of the Fourier transform, its lack of spatial localization, has favored the development of alternative tools for the local analysis of one-dimensional signals and images. The spectrogram technique, Gabor filters and wavelets are introduced with some examples of applications. Also, the importance of the spectrum phase is emphasized.
\end{abstract}

\section{Introduction}

The Fourier transform (FT) has played a very important role in the development of image processing techniques, specifically in the field of microscopic imaging. One of its major contributions was to help in understanding the content of an image in terms of spatial frequencies (low frequencies, mean frequencies and high frequencies). This understanding of the content of an image allowed scientists to imagine how to modify this content, with appropriate filters, in order to restore and improve images. It also allowed them to imagine how to reconstruct three-dimensional objects from a series of two-dimensional filtered images (tomography) as the filling of the three-dimensional frequency space with the information extracted from spectra of two-dimensional images, for periodic objects (crystals) as well as non-periodic (isolated) objects. Thus, the merits of the Fourier transform do not need to be demonstrated. But these merits must not hide the fact that the FT suffers from several limitations. These limitations have (at least) two origins.

The main limitation originates from the definition of the FT, which concerns the decomposition of a signal (with one, two, three... spatial dimensions) into a weighted sum of sinusoids. As anybody knows, a sinusoid is a periodic function with an infinite support, whose period $T$ is inversely proportional to the frequency $f$. Thus, a sinusoid does not possess any spatial localization: it extends over the whole domain of definition. This lack of spatial localization 


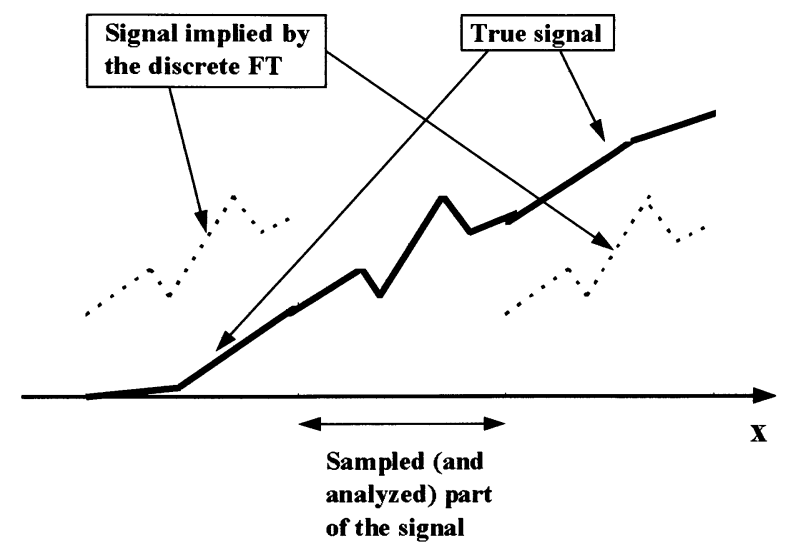

Fig. 1. - Schematic illustration of the problem of windowing a signal for subsequent analysis by the Fourier transform: although the original signal is non-periodic, periodicity is implicitly assumed, which often creates discontinuities at the extremities of the analyzed area.

constitutes the main limitation of the FT: the origin of a particular frequency of the frequency spectrum cannot be defined in the original signal space. Conversely, all the frequencies present in the spectrum must be considered in order to reconstitute the signal at a given position.

A secondary limitation, the nature of which is more technical and less fundamental, comes from the fact that the discrete FT corresponds to a periodization of the signal in the real space, the period being inversely proportional to the frequency sampling rate (in the same way as the discretization of the signal corresponds to a periodization of the frequency spectrum (Fig. 1)). Considering that the real image is in general non-periodic, this "inconsistency" between the real signal and the discrete signal produces artefacts in the computed spectrum and in the signals reconstituted after filtering.

In this paper, our purpose is to put emphasis on these limitations of the FT and to show that some techniques are currently being made available that will allow us to surpass them. In the following section, we mainly consider the technical problems encountered when trying to use the FT for image pre-processing and image analysis, and we discuss some of the solutions which can be used for avoiding them. In the next section, we describe some of the possibilities already developed for surpassing the fundamental limitation of the FT. At the same time, we put emphasis on the importance of the phase spectrum, which appears to have been largely underestimated when considering the spectrum of an image.

Finally, we give our conclusion concerning the question raised in the title of this paper.

\section{Practical Consequences of the Limitations of the Fourier Transform}

\subsection{It is not Possible to Perform Adaptive Image Processing from the FT}

Consider for instance image pre-processing tasks such as:

- contrast enhancement,

- improvement of the signal-to-noise ratio (SNR),

- image restoration (correction of degradations induced by the imaging system). 
What we mean by global (or non-adaptive) processing is a method which does the same work everywhere in the image. In contrast, a local (or adaptive) processing is a method which does not do the same job everywhere (the algorithm takes into account the local content of the image: local contrast, local SNR...).

The Fourier transform (FT) played an important role in the understanding of image formation in imaging systems like the optical microscope (Duffieux [1]) and the transmission electron microscope [2]. As an extension, many authors have suggested improving the images produced by these instruments by following the "reverse path":

$$
\text { image } \stackrel{\text { FT }}{\rightarrow} \text { complex frequency spectrum } \stackrel{\text { filter }}{\rightarrow} \text { filtered spectrum } \stackrel{\mathrm{FT}^{-1}}{\rightarrow} \text { filtered image (\# Object) }
$$

This approach has furnished a preliminary basis to numerous image processing tools (even if, practically, filtering in the frequency space was soon replaced by convolution in the real space). But this approach is also limited. Its limits can be easily identified when considering the nature of the FT. As the FT is the decomposition of a signal (the image grey levels, for instance) into a weighted sum of sinusoids, the resulting image analysis and image processing tools based on the FT can only be global. It means that they work on the whole image without discernment.

If we consider image pre-processing, for instance, (improvement of SNR or contrast enhancement), low-pass filtering (smoothing) or high-pass filtering (enhancement) as they are furnished in commercial image processing software are often insufficient. The reason is that their expected effect is often accompanied by undesired effects (loss of resolution in case of smoothing, degradation of SNR in the case of contrast enhancement). Local image processing tools, able to create a sufficient compromise (smoothing without strong deterioration of resolution; contrast enhancement without strong degradation of SNR) are necessary [3].

\section{Global Pre-Processing}

- Contrast enhancement: since details, edges... constitute the high frequencies of an image, contrast enhancement within the framework of the FT means high-pass filtering. The problem is that noise is also a high-frequency component. Thus, high pass filtering also results in noise increase and in a degradation of SNR!

- SNR improvement: since noise is (essentially) a high-frequency component, SNR improvement within the framework of FT means low-pass filtering. Unfortunately, diminishing the weight of high-frequencies also results in a degradation of contrast and resolution!

\section{Local Pre-Processing Tools}

As written above, these tools have the aim of taking into account the local characteristics of the image (local gradient, local SNR), in order to avoid the drawbacks of the global filtering tools. For instance, it would be useful to smooth image zones where the grey levels vary slowly, but to avoid smoothing zones (like edges, details) where the contrast is high, in order to avoid the deterioration of contrast and resolution. In the same way, it would be useful to perform contrast enhancement near edges and details, but to avoid performing it in zones with a very weak contrast (because in this situation, there is nothing to enhance, except noise).

It should be stressed that these approaches bear some coherence with the human visual system, which is very sensitive to noise in regions with weak contrast, but moderately disturbed by noise present in regions of high contrast. It should also be stressed that preliminary formulations of these ideas can be attributed to Gabor [4], who anticipated that image deblurring should be based on directional sensitive filtering. 


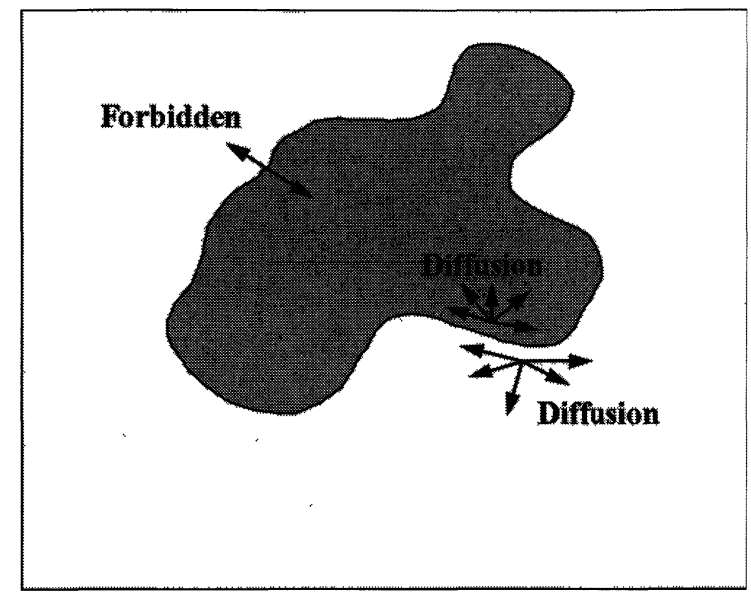

Fig. 2. - Schematic illustration of the anisotropic diffusion process: diffusion (smoothing) is allowed within homogeneous regions of the image but is forbidden across boundaries (i.e. regions with a high grey level gradient).

Although the literature concerning local image processing has begun to be very rich, we will limit ourselves to two examples, one concerning SNR improvement and one concerning contrast enhancement.

\section{Smoothing by Anisotropic Diffusion}

The principle has been stated above: smoothing is allowed within zones with a constant grey level, but is not allowed to diffuse across boundaries (Fig. 2).

This can be obtained by solving a heat diffusion-like equation iteratively [5-8]. A preliminary estimation of the grey levels gradient is computed from the noisy image. Smoothing is performed locally (a weighting coefficient, inversely proportional to the local gradient, is applied). After smoothing, a new (more realistic) estimation of the gradient is computed. Smoothing is applied again, etc. An example is given in Figure 3.

\section{Local Contrast Enhancement}

Several methods for contrast enhancement which take into account local characteristics of images have been suggested $[9,10]$. We have often successfully used the method suggested by Beghdadi and Le Negrate [11]. This method consists of estimating the average grey level of edges in the neighborhood of the pixel (or voxel) to be processed, of defining the local contrast (as a function of the pixel grey level and of this edges grey level), of choosing a function for contrast amplification, and finally of computing the pixel grey level resulting from the amplified contrast. One great advantage of this method is that it leads to a reinforcement of the histogram bimodality (or multi-modality), which is of great usefulness for the purpose of automatic segmentation. One extension of the method allows us to perform contrast enhancement and smoothing simultaneously [12].

Some illustrations of these methods for local image pre-processing can be found in $[3,7,13]$. 


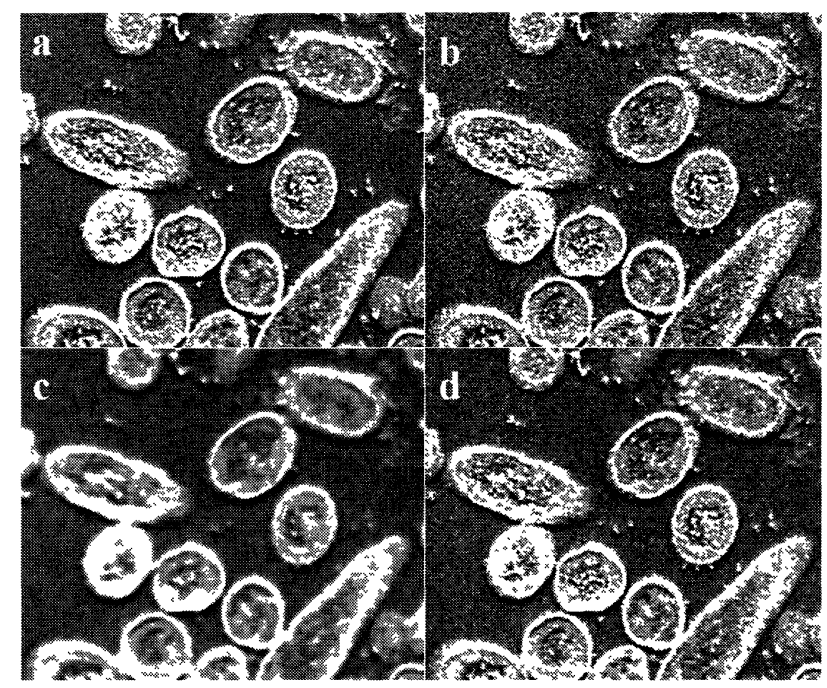

Fig. 3. - Example of SNR improvement by anisotropic diffusion. a) original image, b) noisy image (noise was artificially added), c) result of median filtering $(5 \times 5$ pixels), d) result of smoothing by anisotropic diffusion. The deterioration of resolution is reduced in the latter case.

\subsection{Problems Resulting from the Periodization of the Original Signal}

A non exhaustive list of such problems comprises:

- the artifacts which disturb image analysis in the frequency space.

Discontinuities on the edges (left/right, top/down) of images (see Fig. 1) induce artificial high frequencies which appear as horizontal and vertical lines in the spectrum, not only in the neighborhood of the zero frequency, but also in the neighborhood of high amplitude peaks. These artificial frequencies and associated lines may be sufficiently large as to prevent a precise analysis of these peaks [14].

- the artifacts introduced by filtering performed in the frequency space.

The artificial frequencies introduced by the image periodization will be modified by the filter. As a result, other artifacts will appear when going back to the real space (Gibbs phenomenon). These artifacts are mostly concentrated in the neighborhood of existing discontinuities (regions with high contrast, as edges of objects, for instance).

- the artifacts observed when computing the fractal dimension.

This specific image analysis tool is used more and more often, in near field microscopy as well as in electron or confocal microscopy $[13,15]$. One of the methods used for computing the fractal dimension of an image (and thus of the corresponding object) makes use of the FT [16]: the logarithm of the power spectrum $P(f)$ is first computed; it is then integrated over annuli centred at increasing frequencies $f$. A plot of $\log (P(|f|))$ as a function of $\log (|f|)$ is drawn and the regression coefficient (which is proportional to the fractal dimension) is computed (see Fig. 4). The artifacts mentioned above may disturb this analysis significantly [17].

These problems can be partially avoided: since they all come from the eventual discontinuities on the edges of images, they would disappear if one were able to suppress the discontinuities. This can be done along the following approaches:

a) Windowing: a classical solution consists of limiting the analyzed area by applying a mask whose value decreases smoothly to zero on the edges to the original image. A circular window 


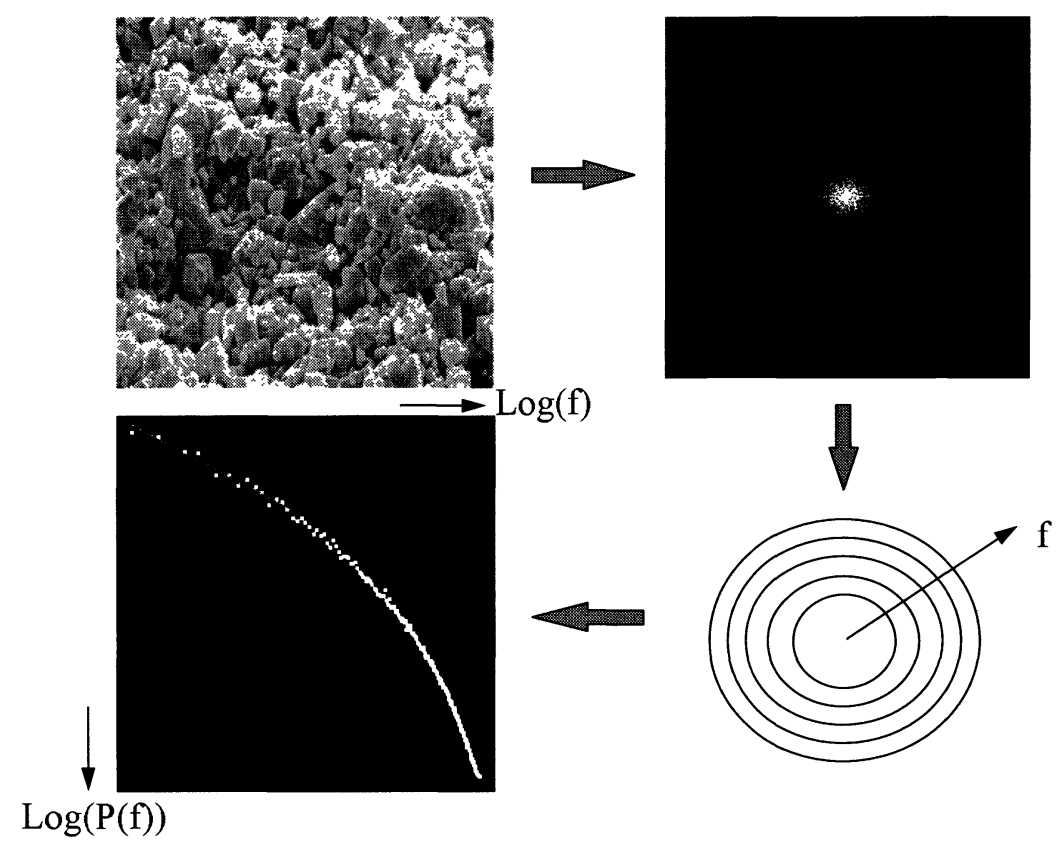

Fig. 4. - Illustration of one of the methods available for computing the fractal dimension of one image. This method, which uses the power spectrum as an intermediate step, is prone to suffer from the artifacts introduced by discontinuities.

is generally applied, in order to restore isotropy. For a detailed analysis of the advantages and drawbacks of different shapes for the window, see [14]. However, with this method, a part of the image is not analyzed.

b) Padding: in order to avoid the previous problem, we can paste the original image onto a larger one. Generally, a double-sized image is used, where the grey level of the added part is often set to the average grey level value of the original image. This method allows us to keep the complete original image, but the computation time is multiplied by a factor of four and the problem of discontinuity is not completely avoided.

c) Mirror effect: a more elegant method consists of doubling the image size (in both directions) and applying a mirror symmetry. This method was originally applied in spectroscopy [18], and is periodically rediscovered $[17,19,20]$. Some of these different possibilities are exemplified in Figure 5.

\section{Extensions of the Fourier Transform}

As we pointed out in the introduction of this paper, the fundamental problem of the FT is that it does not perform a local analysis of the signal, but only a global analysis. A specific value of the frequency spectrum cannot be associated (even approximatively) to a specific position in the real space. This value represents only the average weight of this frequency in the whole image. Conversely, the signal at a specific position in the image potentially contributes to all the frequencies of the spectrum.

This situation is clearly unsatisfactory: in the same way as local image processing tools are necessary, there is a need to perform local signal (image) analysis. 


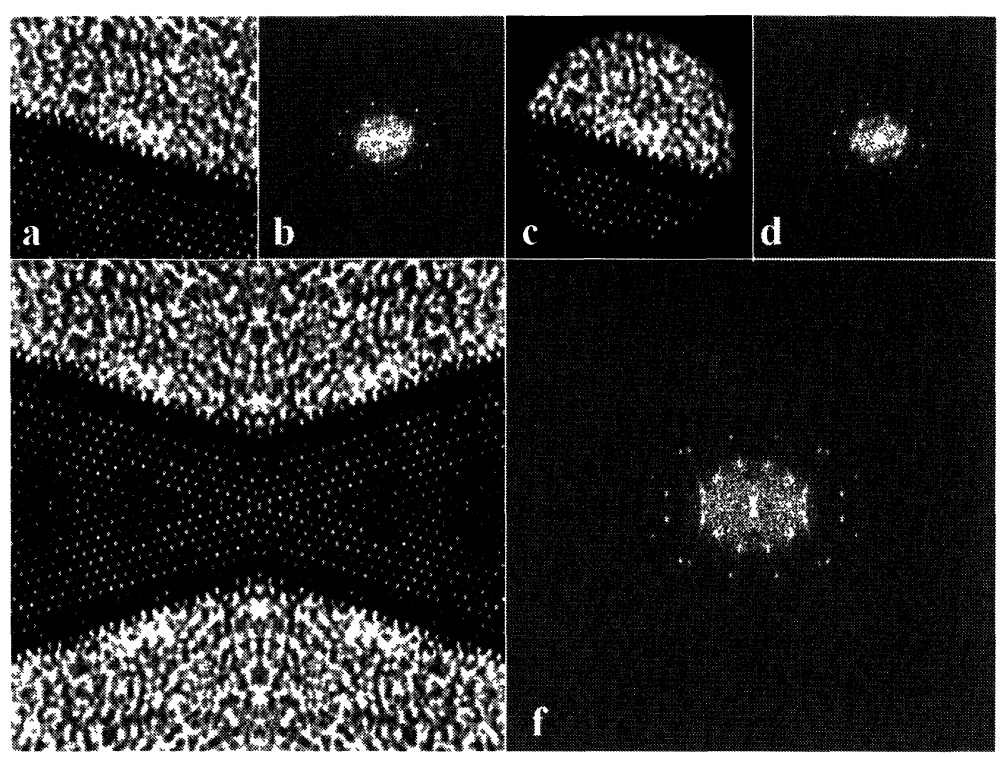

Fig. 5. - Illustration of some possibilities for avoiding the artifacts created in the Fourier space by discontinuities in the real space. a, b) original image (Courtesy of P. Ruterana) and its power spectrum (notice the vertical streaks). c, d) Masked image and its power spectrum (notice the absence of vertical streaks). e, f) Mirrored image and its power spectrum.

As a reaction to the lack of spatial localization of the FT, complementary methods and variants were developed for signal analysis. These methods include: the spectrogram approach, Gabor filters, the Wigner-Ville transformation [21-23] with many variants and wavelets [24,25].

These methods result in a better compromise between spatial localization and frequency localization of phenomena [26,27]. They also allow for multi-scale analysis.

Below, we give a brief description of some of these tools and of the possibilities they provide.

\subsection{Local Fourier Transform (or Windowed FT, or Spectrogram)}

A natural extension of the FT, which allows the partial solving of the problem of spatial localization (or, more generally, of localization in the real space), consists of analyzing the signal "piecewise", by applying a moving window. In order to avoid the problem of discontinuity mentioned above, this window must have smooth edges (Hanning window, Gaussian window...). It is then possible to compute the signal spectrum for different positions of the window covering the signal and to obtain a local spectrum analysis, allowing the characterization of non-stationary phenomena.

For one-dimensional signals, the result obtained is called a spectrogram. It is a kind of image: the horizontal axis corresponds to the spatial coordinate (or time, or wavelength/energy in spectroscopy) and the vertical axis represents the frequency. Hence the terminology "time/frequency representation" which, when transposed to spatially-varying signals becomes "spatial/spatial frequency representation".

An illustration is given in Figure 6, which represents a simulated signal containing three frequencies, the weight of these frequencies varying within four parts of the signal (the weights are 50-20-10/50-20-50/100-20-10/50-50-10, respectively), its power spectrum and its spectrogram. 


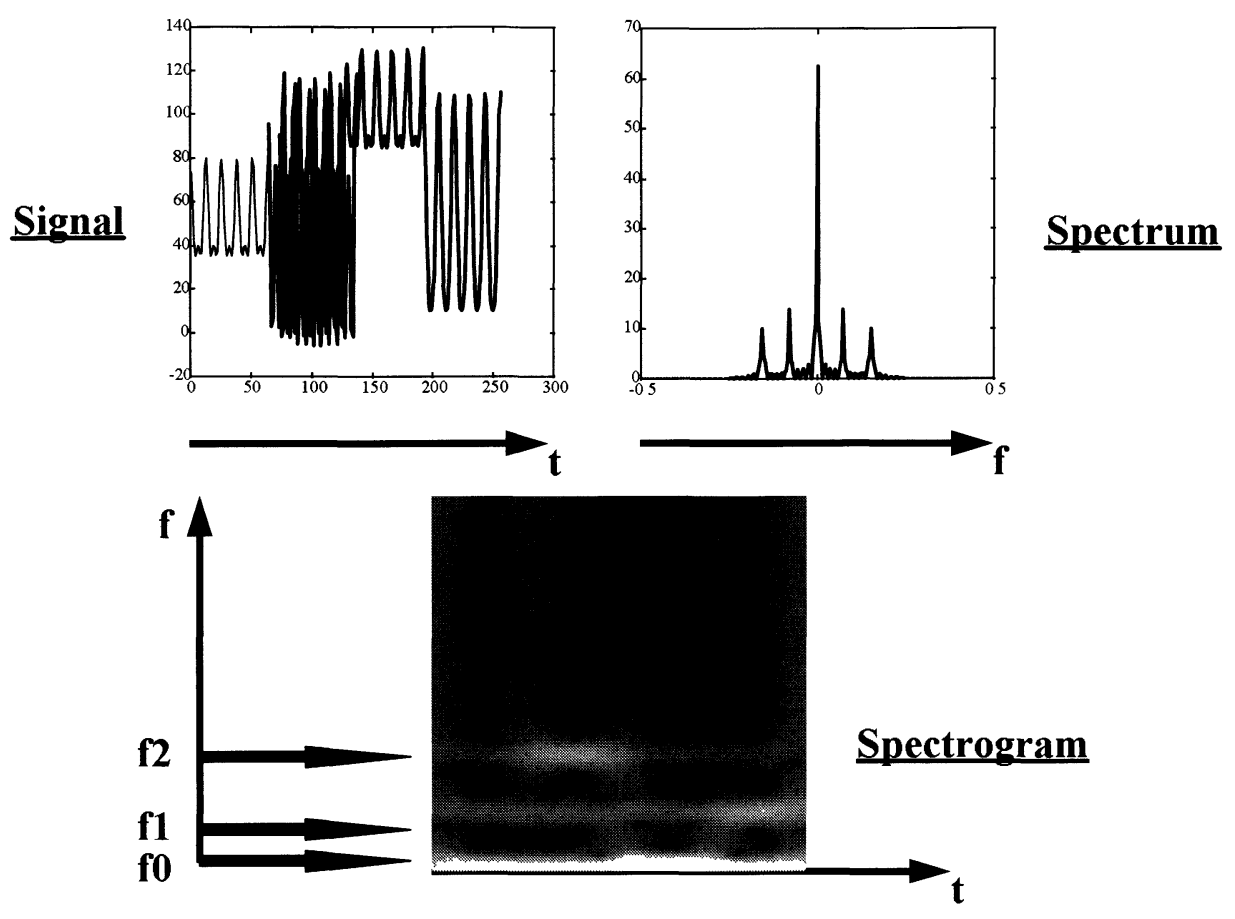

Fig. 6. - Illustration of the spectrogram technique. a) One-dimensional simulated signal. b) Its amplitude Fourier spectrum. c) The spectrogram computed with a Gaussian window (standard deviation $\sigma=15)$. Variations of the weight of the different frequencies $\left(f_{0}, f_{1}\right.$ and $\left.f_{2}\right)$ can be depicted in the spectrogram, but not in the Fourier spectrum.

The spectrogram allows us to observe the change in the frequency content of the signal, while the Fourier transformation only allows us to get the average values.

One can anticipate that such analyses will soon take place in spectroscopies (analysis of fine structures or extended structures, as in EXAFX or EXELFS, for instance).

For two-dimensional signals (images), the same principle can be applied. However, the difficulty is in the representation of the results, which are four-dimensional (two dimensions for the real space and two dimensions for the local reciprocal space). The "object" created resembles the templates introduced in image algebra [28,29]. For this reason, other variants, which produce more compact results, are preferred in this case.

\subsection{Gabor Filters}

As a variant of the spectrogram technique, let us consider the Fourier transform of a modulated and windowed part of a signal $s(x)$. More precisely, let us consider:

- a Gaussian window (standard deviation $\sigma$ )

- a modulation at frequency $f_{0}$.

The signal can now be written:

$$
s^{\prime}(x)=s(x) *\left[G(x, \sigma) \mathrm{e}^{-2 \pi j f_{0} x}\right]
$$




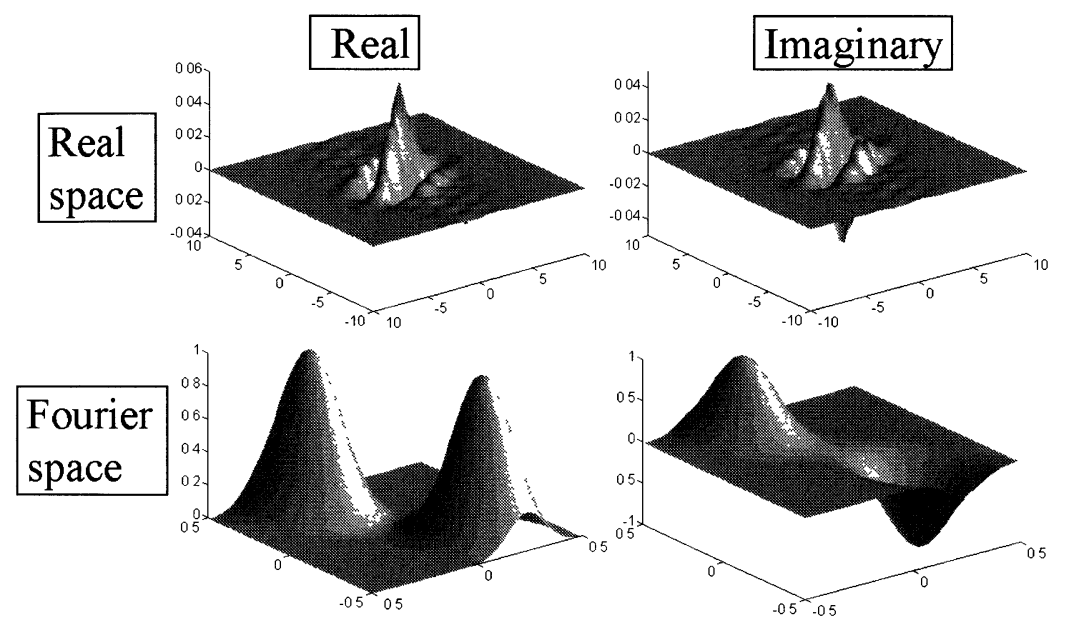

Fig. 7. - Impulse responses (real and imaginary parts) and transfer functions (symmetric and asymmetric parts) of a Gabor filter.

where $*$ represents the convolution operator. $s^{\prime}$ is a complex signal (modulated by a cosine and a sine function at frequency $f_{0}$ ), with a limited extension in the real space.

It can easily be shown that the new signal is equivalent to the original signal filtered (in the frequency space) by two band-pass filters with Gaussian transfer functions (central frequency: $f_{0}$ ), one of them being symmetrical and the other one being anti-symmetrical (Fig. 7). (Note: if these two (symmetrical and anti-symmetrical) filters are added, one ends with the a posteriori equivalent of single-side band (SSB) holography [30-32]).

This kind of filtering provides a good localization in the real space (defined by the value of $\sigma$ ) and in the reciprocal space (bandwidth inversely proportional to $\sigma$ ). It can be shown that the Gaussian window results in the best compromise, in the sense of the uncertainty principle $(\Delta x \Delta f$ is minimum).

Instead of one single Gabor filter, one can use a bank of such filters (set of filters with different values for $f_{0}$ ). This bank of Gabor filters allows us to analyze a signal locally. In the case of images, one must also have different filters with various orientations. One must define a configuration of the filters set so that the whole reciprocal space is approximately covered, without too much overlapping of the transfer functions (generally, a progression of one octave or one half octave, and 4 to 8 orientations, are sufficient). Figure 8 shows the frequency space covering obtained with the so-called "daisy petal" configuration.

The filtered images obtained with these different complex filters may be combined, in order to obtain, for each pair of filter, a filtered amplitude and a phase. We put emphasis on the fact that the image phase information was neglected for a long time, but has begun to be exploited. From our point of view, this information is very important ( $c f$. appendix) and is susceptible, when correctly exploited, to furnish really new results in image analysis.

Some examples of the use of Gabor filters are:

- Automatic stereoscopy: the automatic determination of the third dimension $(z)$ from two stereoscopic two-dimensional $(x, y)$ images must rely on the local estimation of the parallax $(\Delta x)$. One can show that this local parallax is coded within the phase difference between 


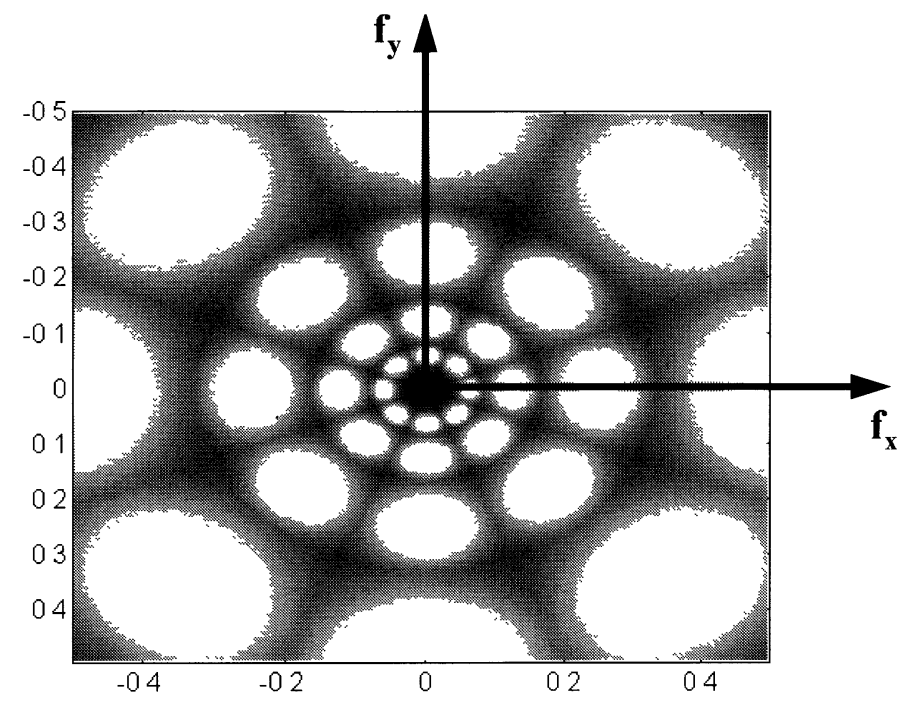

Fig. 8. - Coverage of the frequency space by a bank of Gabor filters in the daisy petal configuration (4 frequencies, 4 orientations). The grey levels are proportional to the amplitude of the transfer functions.

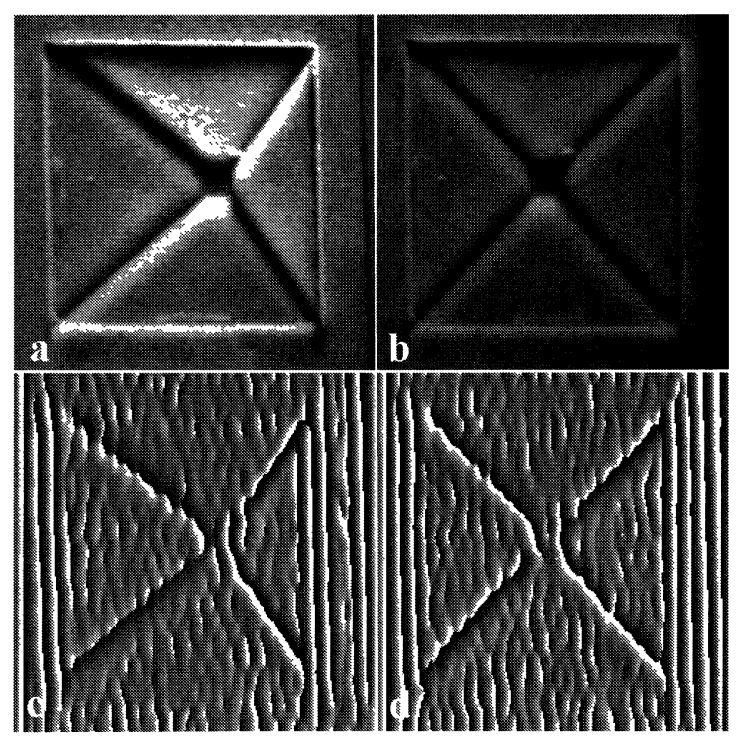

Fig. 9. - Partial illustration of one procedure for automatic computation of the stereo disparity. a, b) Two stereoscopic views of etchpits recorded in Scanning Electron Microscopy (courtesy of S. Simov, Institute of Solid State Physics, Sofia). c, d) The corresponding horizontal phase images obtained via Gabor filtering. The next step consists of cross-correlating these two phase images in order to get the local disparity, and thus the local height. 


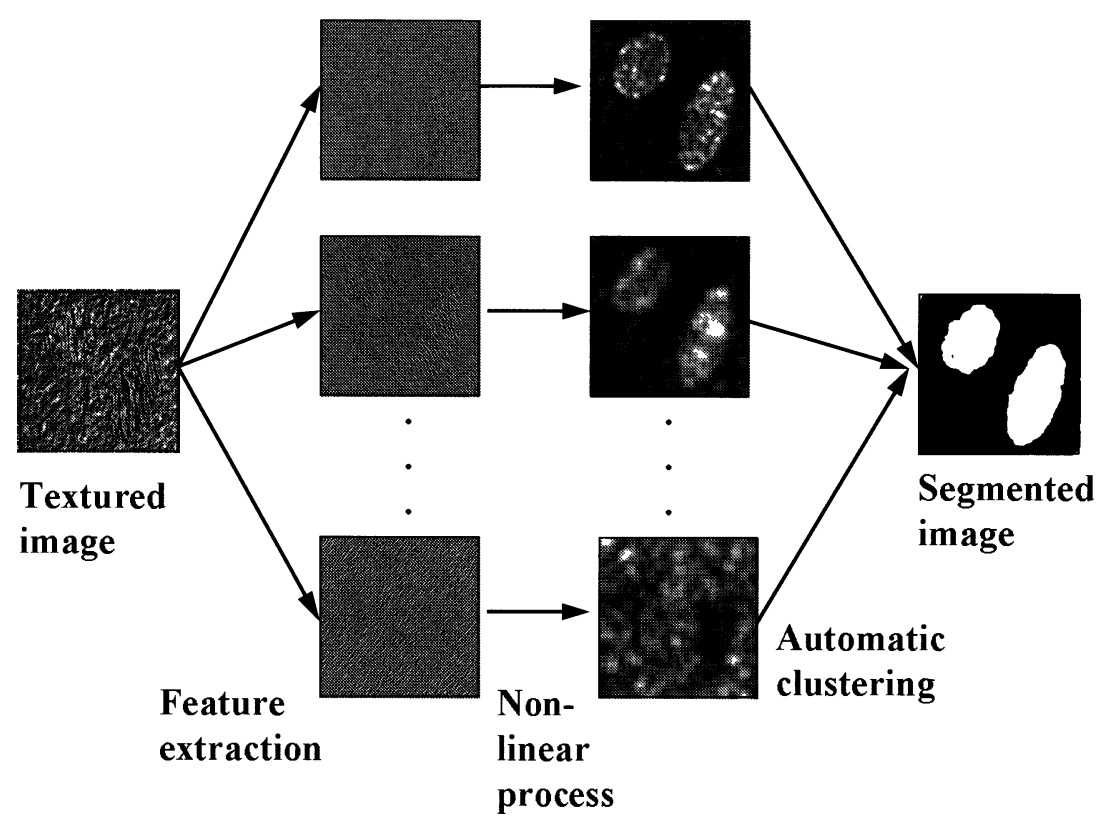

Fig. 10. - Schematic illustration of the process of textured image segmentation: different texture features are extracted using a bank of Gabor filters (only some of them are shown). As a result, each pixel is described by a feature vector. Automatic grouping of similar feature vectors into classes is then performed and a texture class label is provided.

the two images, computed at a frequency which corresponds to the size of the observed details (Bonnet, in preparation). Figure 9 gives an example of the horizontal phase obtained through Gabor filtering for a stereoscopic pair of etchpits.

- Detection and characterization of nanocrystals in high resolution electron microscopy [33], and analysis of dislocations and other defects [34,35].

- Recognition and segmentation of textured images [36,37]. A schematic illustration of the process is given in Figure 10.

- Analysis of object movements in video-microscopy (optical flow technique) [38].

\subsection{Wavelets}

The Fourier transform results in the decomposition of a signal into a set of basis functions (sinusoids) which can be characterized by a perfect frequency localization but a total lack of spatial localization. The idea of wavelets is to decompose a signal into a set of basic functions which possess a certain number of properties allowing a local analysis:

- a good localization in the spatial domain (more generally: in the real space) and in the frequency domain (the reciprocal space) simultaneously (we have already mentioned, with Gabor functions, that this is not utopian),

- if possible, an orthogonality property (the scalar product between two basic functions is null). This property guarantees the absence of the redundancy of the decomposition,

- the basic functions can be deduced from one of them by simple dilation/contraction,

- the reverse transformation is possible (one can go back to the original space after decomposition and modification of the weights of the different components, as with the inverse Fourier transformation). 
FT Spectrogram Wavelet
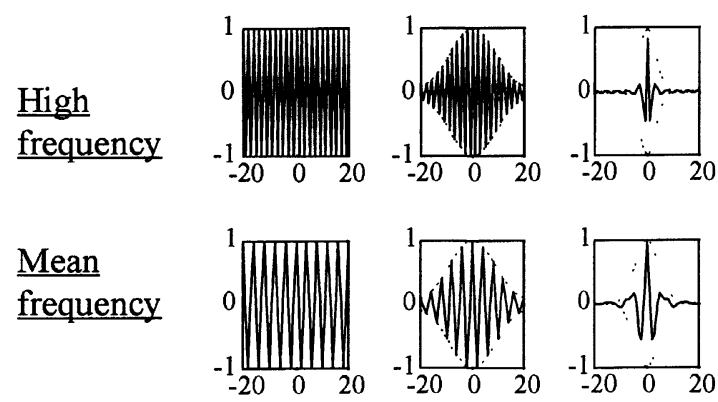

$\underline{\text { Low }}$ frequency
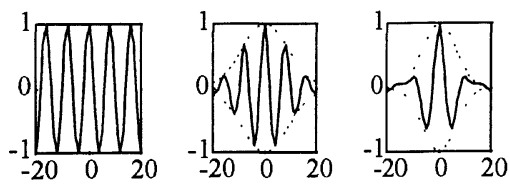

Fig. 11. - Schematic representation of the analyzing signals for the classical Fourier transform, the windowed Fourier transform (spectrogram) and wavelets.
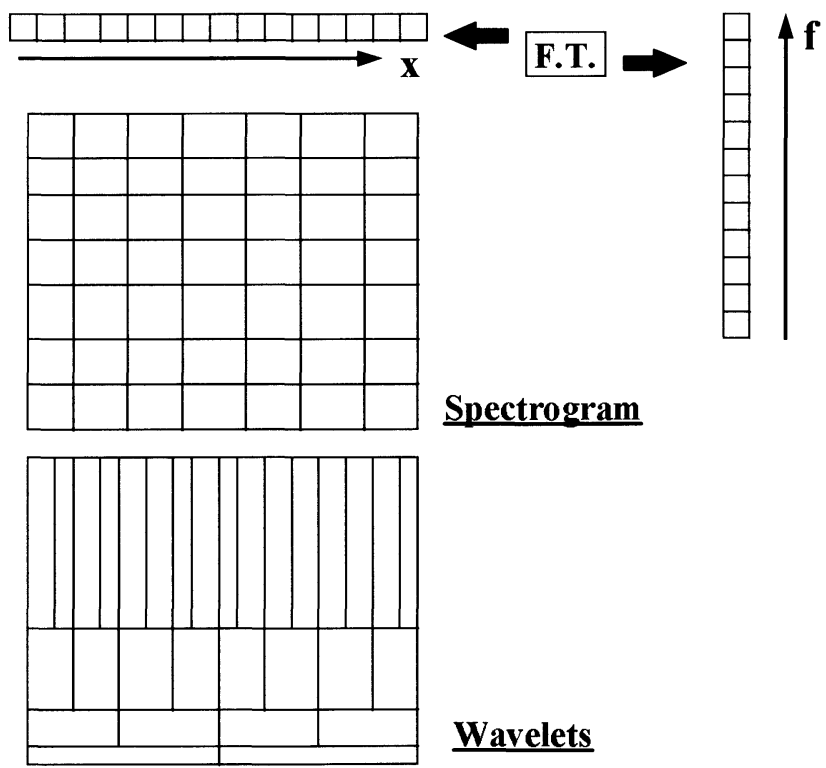

\section{$\underline{\text { Spectrogram }}$}

\section{Wavelets}

Fig. 12. - Schematic comparison of the real space $(x)$ and frequency space $(f)$ coverages for the classical Fourier transform, the windowed Fourier transform (spectrogram) and wavelets.

The difference between the decomposition into wavelets and the local Fourier transform technique is illustrated in Figure 11. With the local FT, the window size is maintained constant. But the modulation frequency varies. The result is that the analyzing signal varies with the frequency. In a wavelet decomposition, the analyzing signal (the wavelet) is of constant shape. 


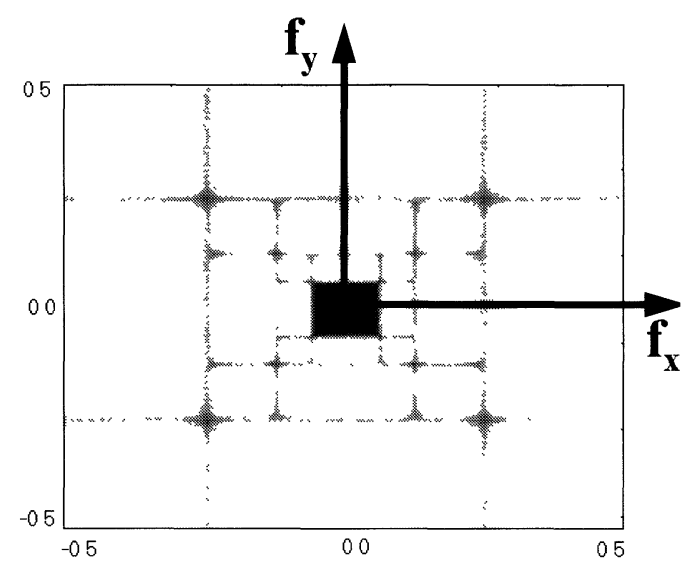

Fig. 13. - An example of frequency space coverage with wavelets. The wavelets used are B-spline wavelets [41].
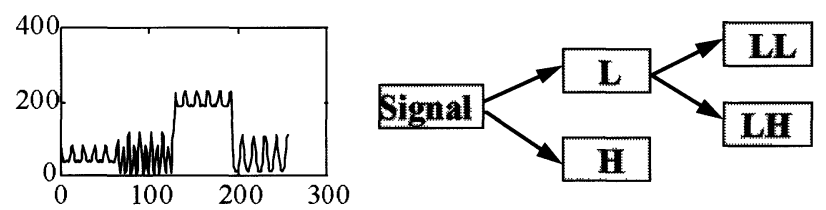

Original signal

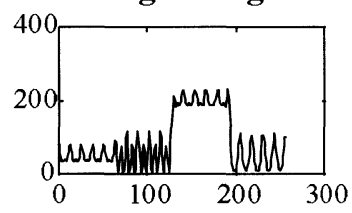

Low
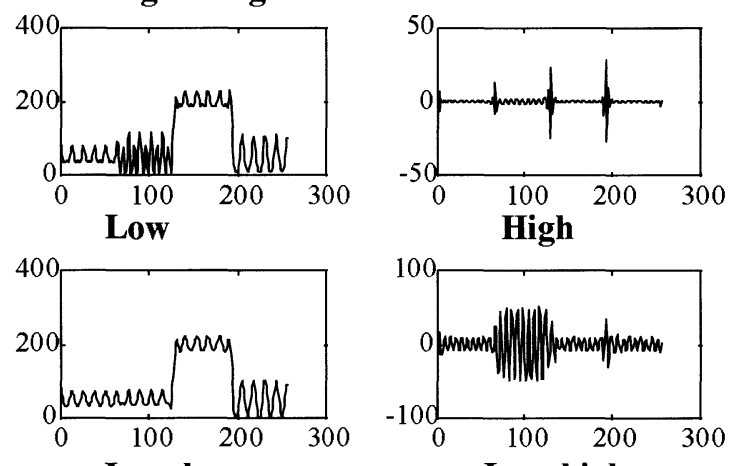

Low-low

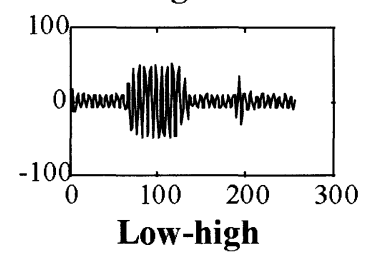

Fig. 14. - Illustration of the wavelet decomposition of a simulated one-dimensional signal. The original signal could be reconstituted by adding up the low frequency component (Low- low) and the high frequency components (High + Low-high). But what is more useful is to modify selectively some components for performing "intelligent" smoothing, enhancement or restoration. The main difference with classical Fourier filtering is that low and high frequencies are spatially localized in the case of wavelets.

But the size of the analyzed signal (envelope of the wavelet) varies with the frequency. This is more logical: in order to get the characteristic information, we need to analyze a larger zone of the signal at low frequency than at high frequency.

From this, the resulting reciprocal space covering is different (Figs. 12, 13).

Figure 14 illustrates the wavelet decomposition of a 1D signal (the same as in Fig. 6a) while figure 15 illustrates the wavelet decomposition of an image (the same as in Fig. 3a). 


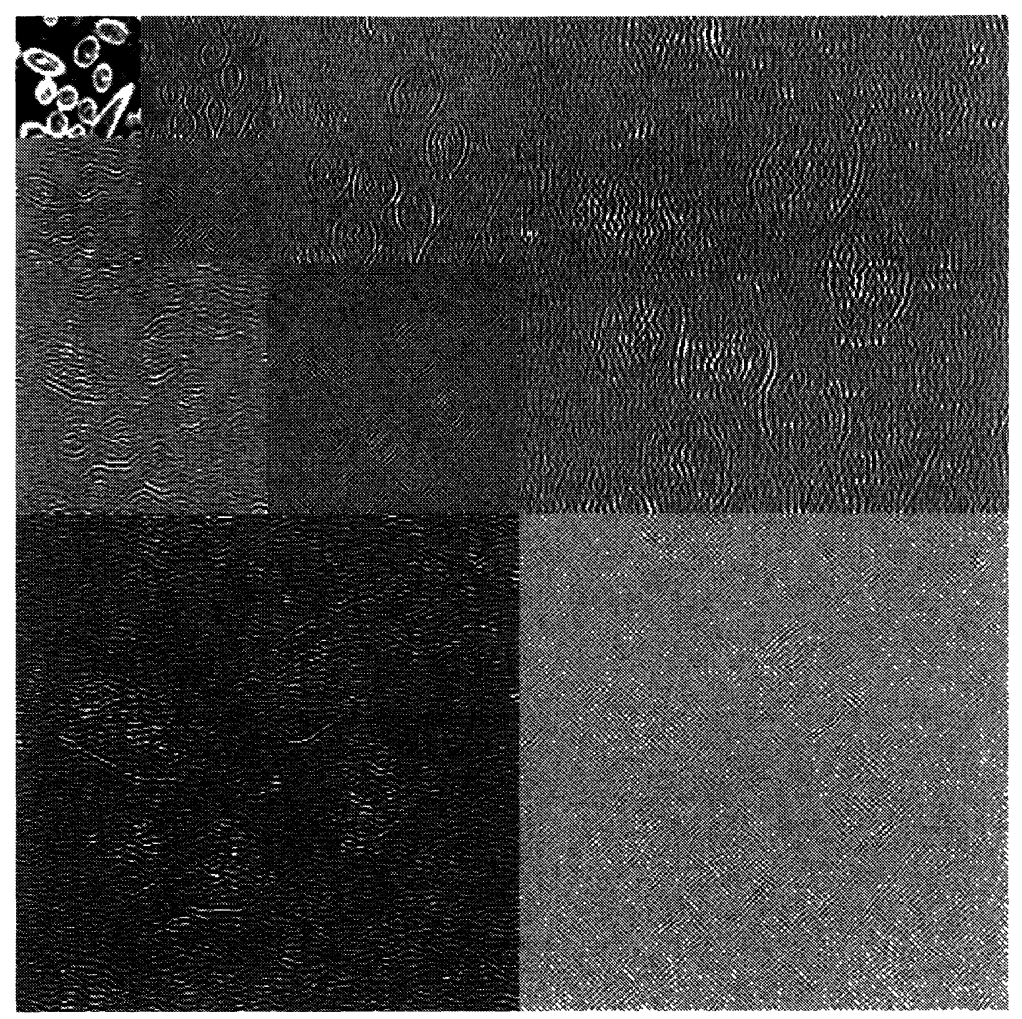

Fig. 15. - Same as Figure 14, but the signal is an image (the original image is that of Fig. 3a). (a) Low frequency component. High frequency components can be split into vertical (b), horizontal (c) and diagonal (d) components. Three stages of the decomposition are shown here. When more levels of decomposition are needed, the low frequency component is further split. In the case of wavelet packets (not discussed in the text), the high frequency components are also decomposed (see [36] for more details).

Although the application of wavelets in microscopic imaging has been very restricted (see however $[39,40]$ ), they would permit a very efficient multi-resolution (multi-scale) analysis of signals and images. One can anticipate that, as in many other fields of imaging, they will also be used for:

- SNR improvement (local and multi-scale processing),

- local contrast enhancement,

- image restoration,

- image classification,

- data fusion (combination of information originating from different imaging techniques).

\section{Conclusion}

Certainly, the Fourier transform is not dead. It still has a future in the community of image processing. First, it should be stressed that its usefulness as a calculus tool (for computing correlation or convolution, for instance) is out of discussion. Second, many possibilities have 
a)

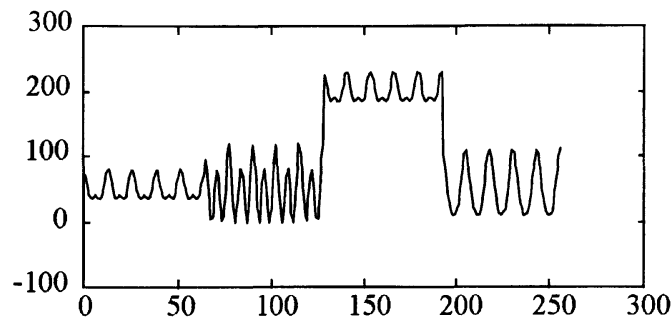

b)

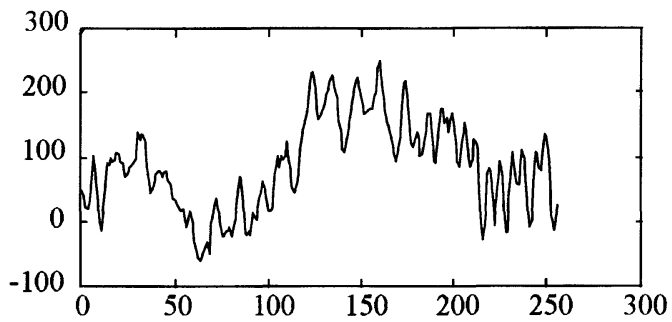

Fig. 16. - Illustration of the importance of the phase spectrum. The two signals ( $a$ and $b$ ) have the same amplitude spectrum. They differ only by their phase spectrum.

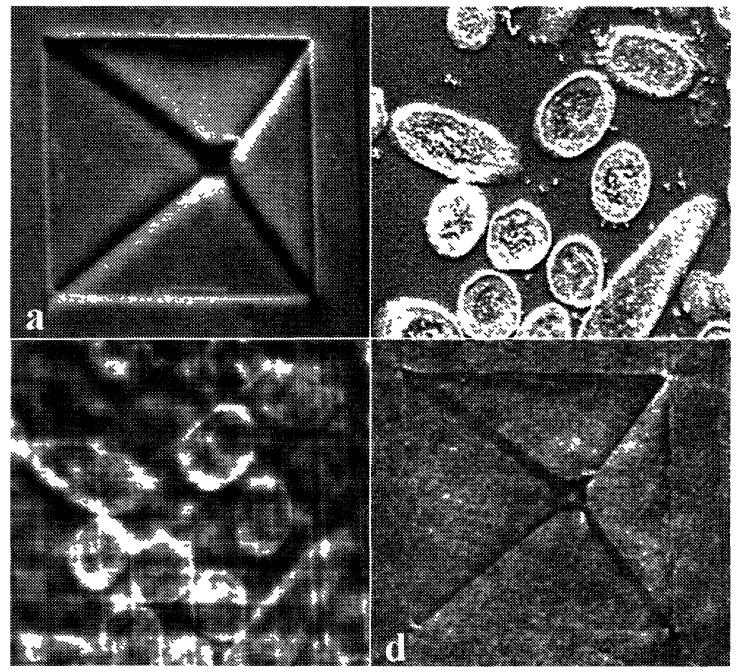

Fig. 17. - Illustration of the importance of the phase spectrum. (c) was built by combining the amplitude spectrum of (a) and the phase spectrum of (b). The reverse was made for (d). From the resemblance of (c) with (b) (and (d) with (a)), it can be concluded that the role played by the phase spectrum is greater than the role played by the amplitude, in this specific but reproducible case.

yet to be explored, such as the fractional Fourier transform, for instance. More specifically, we consider that the information contained in the phase spectrum has been largely underestimated, compared to the amplitude (or intensity) spectrum. However, the information contained in the spectrum phase is at least as important as that contained in the amplitude spectrum ( $c f$. appendix). Recent results obtained by holographic methods demonstrate many potentialities of studying the phase. But holography is not always necessary. Phase information is partially 
coded in images. The problem is to decode it. Once we are able to compute the local phase (we have seen that Gabor filters and wavelets can help to do it), it remains to develop specific tools in order to extract pertinent information from it (problem of phase unwrapping for instance).

But, in addition to the Fourier transformation, other tools are becoming available, such as wavelets for instance. These tools will probably replace the primary role of the Fourier transformation, for which the spatial localization is very insufficient.

\section{Appendix}

\section{On the Importance of Phase in the Representation of Signals}

This importance can easily be demonstrated through a few observations and computer experiments:

a) two very different signals may have the same amplitude spectrum (they differ by their phase spectrum), as can be seen in Figure 16 (the common amplitude spectrum is displayed in Fig. 6b).

b) consider two images: $I_{1}$ and $I_{2}$. Let us compute their amplitude spectra $\left(A_{1}\right.$ and $A_{2}$, respectively) and their phase spectra $\left(\mathrm{P}_{1}\right.$ and $\mathrm{P}_{2}$, respectively). Let us reconstitute two images $\left(\mathrm{I}_{1}^{\prime}\right.$ and $\left.\mathrm{I}_{2}^{\prime}\right)$ after mixing the spectra $\left(\mathrm{A}_{1} \cdot \exp \left(j \mathrm{P}_{2}\right) \rightarrow \mathrm{I}_{1}^{\prime} ; \mathrm{A}_{2} \cdot \exp \left(j \mathrm{P}_{1}\right) \rightarrow \mathrm{I}_{2}^{\prime}\right)$. In general (for non periodic objects), $I_{1}^{\prime}$ is closer to $I_{2}$ than to $I_{1}$ and $I_{2}^{\prime}$ is closer to $I_{1}$ than to $I_{2}$ (see Fig. 17). This proves that the phase information is more important than the amplitude information.

\section{References}

[1] Duffieux P.M., L'intégrale de Fourier et ses applications à l'Optique (see the Introduction of this special issue for more references).

[2] Hawkes P.W., in "Computer Processing of Electron Microscope Images", P.W. Hawkes, Ed. (Springer-Verlag Berlin, 1980) pp. 1-33.

[3] Bonnet N., in "Handbook of Microscopy. Applications in Material Science, Solid-State Physics and Chemistry" S. Amelincks, D. Van Dyck, J. Van Landuyt and G. Van Tendeloo, Eds. (VCH, 1996) Methods II, pp. 923-952.

[4] Gabor D., Lab. Invest. 14 (1965) 63.

[5] Perona P. and Malik J., IEEE Trans. PAMI 12 (1990) 629.

[6] Gerig G., Kikinis R., Kubler O. and Jolez F.A., IEEE Trans. Med. Imag. 11 (1992) 221.

[7] Vautrot P. and Bonnet N., Proc. 13th Int. Conf. Electron Microsc. (ICEM13) Paris 1 (1994) 429.

[8] Biswas S., Pal N.R. and Pal S.K., Pattern Recognition 29 (1996) 497.

[9] Pizer S.M., Amburn E.P., Austin J.D., Cromartie R., Geselowitz A., ter Haar Romeny B., Zimmerman J.B. and Zuiderveld K., Comp. Vision, Graph. Image Proc. 39 (1987) 355.

[10] Paranjape R.B., Morrow W.M. and Rangayyan R.M., CVGIP: Graph. Models Im. Proc. 54 (1992) 259.

[11] Beghdadi A. and Le Negrate A., Comp. Vision Graph. Image Proc. 46 (1989)162.

[12] Le Negrate A., Beghdadi A and Dupoisot H., CVGIP: Graph. Models Im. Proc. 54 (1992) 13.

[13] Bonnet N., Lucas L. and Ploton D., Scanning Microsc. 10 (1996) 85. 
[14] Saxton O., Proc. 15th Pfefferkorn Conference (Electron image and signal processing). SilverBay (1996).

[15] Bonnet N., Dolidon F., Diallo B., Durand C. and Herbin M., Proc. 13th Int. Conf. Electron Microsc. (ICEM13) Paris 1 (1994) 445.

[16] Pentland A.P., IEEE Trans. PAMI 6 (1984) 661.

[17] Aguilar M., Anguiano E. and Pancorbo M., J. Microsc. 172 (1993) 233.

[18] Spence J., Ultramicrosc. 4 (1979) 9.

[19] Vicario E., Doghmane N. and Tholomier M., J. Microsc. Spectrosc. Electron. 14 (1989) 95.

[20] Anguiano E. and Aguilar M., Proc. 13th Int. Cong. Electron Microsc. (ICEM13) Paris 1 (1994) 433.

[21] Martin W. and Flandrin P., Signal Proc. 8 (1985) 215.

[22] Zhu Y.M., Peyrin F. and Goutte R., Ann. Télécomun. 42 (1987) 105.

[23] Zhu Y.M., Peyrin F., Goutte R. and Amiel M., Traitement du Signal 9 (1992) 281.

[24] Meyer Y., Les ondelettes. Algorithmes et applications (Armand Colin, 1993).

[25] Burke Hubbard B., Ondes et ondelettes. La saga d'un outil mathématique (Pour La Science Diffusion Belin, 1995).

[26] Bovik A.C., Clark M. and Geisler W.S., IEEE Trans. PAMI 12 (1990) 55.

[27] Reed R. and Wechsler H., IEEE Trans. PAMI 12 (1990) 1.

[28] Hawkes P.W., Optik 98 (1995) 81.

[29] Hawkes P.W., Microsc. Microanal. Microstruct. 6 (1995) 159.

[30] Gabor D.J., Inst. Elec. Eng. 93 (1946) 429.

[31] Wade R.H., in "Computer Processing of Electron Microscope Images", P.W. Hawkes, Ed. (Springer-Verlag Berlin, 1980) pp 223-255.

[32] Bonnet N., in "Microscopie Electronique en Science des Matériaux" B. Jouffrey, A. Bourret et C. Colliex , Eds (Editions du CNRS, 1983) pp. 75-84.

[33] Hÿtch M. and Gandais M., Philos. Mag. A 72 (1995) 619.

[34] Hÿtch M., Proc. 15th Pfefferkorn Conference (Electron image and signal processing). SilverBay (1996).

[35] Hÿtch M., Microsc. Microanal. Microstruct. 8 (1997) 41-57.

[36] Vautrot P., Segmentation et classification d'images texturées par filtrage spatio-fréquentiel: ondelettes splines et filtres de Gabor, PhD Thesis. University of Reims, France (1996).

[37] Vautrot P., Bonnet N. and Herbin M., Proc. IEEE Int. Conf. Image Processing, Lausanne (1996)

[38] Remolu A. and Bonnet N., Proc. 15ème Colloque GRETSI Juan-les Pins (1995) 1229.

[39] Gomez A., Beltran del Rio L., Romeu D and Yacaman M., Scanning Microscc. Suppl. 6 (1992) 153.

[40] Livens S., Scheunders P., Van de Wouwer G., Van Dyck D., Smets H., Winkelmans J. and Bogaerts W., Microsc. Microanal. Microstruct. 7 (1996) 1.

[41] Unser M. and Aldroubi A., in "Wavelets: a tutorial in theory and applications", C.K. Chui, Ed. (Academic Press, 1992) pp. 91-122. 